\title{
Dieta de Sula leucogaster Boddaert (Sulidae, Aves), nas Ilhas Moleques do Sul, Florianópolis, Santa Catarina, Brasil
}

\author{
Joaquim O. Branco ${ }^{1}$, Hélio A. A. Fracassoํ, Irecê F. Machado', Marcos S. Bovendorp ${ }^{1}$ \& José R. Verani ${ }^{2}$ \\ ${ }^{1}$ Centro de Ensino em Ciências Tecnológicas da Terra e do Mar, Universidade do Vale do Itajaí. Caixa Postal 360, \\ 88301-970 Itajaí, Santa Catarina, Brasil. E-mail: branco@univali.br \\ ${ }^{2}$ Departamento de de Ecologia e Biologia Evolutiva, Universidade Federal de São Carlos. Caixa Postal 676, 13565-905 São \\ Carlos, São Paulo, Brasil.
}

\begin{abstract}
Diet of Sula leucogaster Boddaert, in the Moleques do Sul Island, Florianópolis, Santa Catarina, Brazil. Sula leucogaster (Boddaert, 1783) breeds in some coastal and oceanic islands of Brazil, and uses as food a great diversity of prey, captured in flat diving, between 10 to $15 \mathrm{~m}$, beyond ictiofauna discarded in shrimps fisheries. This work aims to supply basic information on the natural feeding of the Brown Booby, through the natural regurgitation collected between 2000 to 2004, in the Moleques do Sul Islands. It was registered the occurrence of 35 species-prey in 257 pellets, totalizing $26,5 \mathrm{~kg}$. Despite the strong seazonality in the diet of $S$. leucogaster, the biggest frequencies of regurgitations had been gotten in the summer, where the Sciaenidae, Engraulidae and Batrachoididae were the more explored prey.
\end{abstract}

KEY WORDS. Brown booby, natural feeding, seabirds.

RESUMO. Dieta de Sula leucogaster Boddaert (Sulidae, Aves), nas Illhas Moleques do Sul, Florianópolis, SC. Sula leucogaster (Boddaert, 1783) nidifica em várias ilhas costeiras e oceânicas do Brasil, e utiliza como alimento uma grande diversidade de presas, capturadas em mergulhos rasos, entre 10 a $15 \mathrm{~m}$, além da ictiofauna descartada na pesca de camarões. Este trabalho teve como objetivo fornecer informações básicas sobre a alimentação natural do atobá-marrom, através dos regurgitos coletados entre os anos de 2000 a 2004, nas Ilhas Moleques do Sul. Foram registradas a ocorrência de 35 espécies-presas em 257 regurgitos, totalizando $26,5 \mathrm{~kg}$. Apesar da marcante sazonalidade na dieta de $S$. leucogaster, as maiores freqüências de regurgitos foram obtidas no verão, onde os peixes Sciaenidae, Engraulidae e Batrachoididae foram as presas mais exploradas.

PALVRAS CHAVE. Alimentação natural, atobá-marrom, aves marinhas.

As aves marinhas constituem um grupo diversificado de espécies que se adaptaram com grande eficiência ao meio marinho, utilizando, principalmente cardumes de peixes pelágicos, lulas e crustáceos como fonte de alimento (FurNESS \& MONAGHAN 1987). O papel destas aves no fluxo de energia através dos ecossistemas marinhos tem sido considerado desprezível, mas estudos recentes demonstraram a sua importância como predadores de topo de cadeia (Furness \& CoOper 1982, CAIRns 1987, 1992, MontevecCHI et al. 1988, Ово 1996). Assim, esse grupo pode ser utilizado como modelo no monitoramento da biodiversidade nesses ambientes (Furness \& CAMPHUYSEN 1997).

Em geral, estudos envolvendo alimentação de aves marinhas têm sido conduzidos durante a época de reprodução, baseados nas presas carreadas para as colônias (BALTZ \& MorejoHN 1977, Diamond 1983, Garthe et al. 1996, Stratoudakis 1999). No Brasil, apesar do extenso litoral, onde 18 espécies nidificam no ambiente insular (BRANCO 2004), existem poucas informações disponíveis sobre a dieta dessas aves (Bege \& Pauli 1989, ВотH \& Freitas 2001, Branco 2001, Schulz-Neto 2004, Alves et al. 2004a, Coelho et al. 2004, KRUL 2004).
O atobá-marrom Sula leucogaster (Boddaert, 1783), nidifica em várias ilhas costeiras e oceânicas do Brasil, desde Santa Catarina até os Penedos de São Paulo e São Pedro, incluindo os arquipélagos de Fernando de Noronha, Abrolhos e Atol das Rocas (Sick 1997, Schulz-Neto 2004, Both \& Freitas 2004). Essa espécie utiliza uma grande diversidade de presas, capturadas em mergulhos rasos, entre 10 a 15m (NeLSON 1978), além da ictiofauna descartada na pesca de camarões (Branco 2001).

Este trabalho teve como objetivo fornecer informações básicas sobre a alimentação natural de Sula leucogaster, através dos regurgitos coletados nas Ilhas Moleques do Sul.

\section{MATERIAL E MÉTODOS}

Os bolos alimentares foram obtidos através de regurgitos espontâneos de S. leucogaster (Both \& Freitas 2001, Alves et al. 2004b), durante as contagens dos ninhos, biometria dos ovos, exemplares e no deslocamento através da colônia da ilha maior do Arquipélago Moleques do Sul (2751's, $\left.48^{\circ} 26^{\prime} \mathrm{W}\right)$, considerado o principal sítio de nidificação das aves marinhas no litoral de Santa Catarina (BRANCO 2004), entre os anos de 2002 
a 2004, sendo obtidos em $2000(n=23), 2001(n=68), 2002$ $(n=58), 2003(n=65), 2004(n=43)$ regurgitos. Esses foram acondicionados em sacos plásticos etiquetados e conservados em caixa térmica com gelo, sendo registrada a idade dos atobás como jovens e adultos, em função das suas plumagens (DORWARD 1962).

No laboratório, as amostras foram identificadas ao menor nível taxonômico possível, de acordo com Menezes \& Figueiredo (1980, 1985), Figueiredo \& Menezes (1978). Em cada regurgito foram registrados o número de presas, comprimento total, biomassa e seu modo de vida. Os itens que não puderam ser determinados, devido ao elevado grau de digestão, foram considerados como bolo alimentar, tendo a sua biomassa total registrada.

O índice de importância relativa (IRI) foi utilizado para avaliar a importância das presas na dieta de $S$. leucogaster, através da equação: $I R I=(\% N+\% P) \% F$, onde $(N)$ freqüência numérica, $(\mathrm{P})$ freqüência de biomassa e $(\mathrm{F})$ freqüência de presas (PINKAs et al. 1971).

\section{RESULTADOS}

Durante o período de 2002 a 2004 foram coletados 257 regurgitos, sendo 222 de adultos e 35 de jovens, totalizando 22,788 e 3,961 kg, com uma média de 1,5 e 1,4 espécies presas e 3,2 e 3,3 itens, respectivamente (Tab. I). Os comprimento médio das presas consumidas pelos adultos foi de $13,1 \pm 4,4 \mathrm{~cm}$ e com uma biomassa de 29,0 $\pm 24,8 \mathrm{~g}$, enquanto que nos itens ofertados aos jovens, o tamanho e a biomassa foram relativamente menores, $11,8 \pm 3,2 \mathrm{~cm}$ e $24,6 \pm 20,2 \mathrm{~g}$ (Tab. I).

Foram registradas a ocorrência de 35 espécies, distribuídas em 32 gêneros e 22 famílias na dieta de $S$. leucogaster (Tab. II). Dessas presas, as classificadas como demersais representaram 50,0\% das espécies amostradas nos regurgitos e contribuíram com $57,1 \%$ da biomassa total carreada à colônia, seguidas das bentônicas com $26,5 \%$ e 7,4\%, e das pelágicas com 23,5\% e $13,7 \%$, respectivamente (Tab. II).

Os peixes Sciaenidae contribuíram com a maior diversidade de presas e biomassa, esse domínio foi corroborado com a aplicação do índice de importância relativa (IRI), onde Paralonchurus brasiliensis (Steindachner, 1875), seguido do Engraulidae Cetengraulis edentulus (Cuvier, 1829), Isopisthus parvipinnis (Cuvier, 1830) e Cynoscion striatus (Cuvier, 1829), destacaram-se como espécies de fundamental importância na dieta do atobá-marrom (Tab. II). O bolo alimentar representou $19,0 \%$ da biomassa total, enquanto que as 21 espécies com IRI menor que 1,0, contribuíram com apenas 7,3\% da biomassa.

Analisando a dieta de $S$. leucogaster, por estação do ano (Fig. 1), verifica-se que ocorreram flutuações sazonais na freqüência e biomassa média de regurgitos carreados à colônia durante o ano, com as maiores contribuições nos meses de verão, seguido de redução brusca no outono e recuperação no inverno, para posterior queda na primavera.

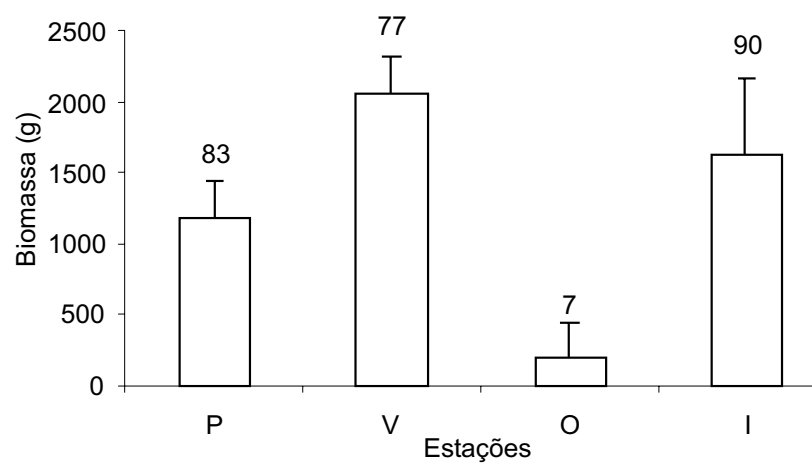

Figura 1. Biomassa média de presas nos regurgitos de $S$. leucogaster, nas Ilhas Moleques do Sul ao longo das estações do ano. As barras indicam erro da média e os valores o número de regurgitos analisados.

\section{DISCUSSÃO}

A diversidade e abundância de presas na dieta de $S$. leucogaster, oscila em função do sítio de nidificação e da atividade de pesca na região. Assim, a importância dos peixes-voadores na dieta dessa espécie pode ser atribuída à sua abundância nas proximidades dos arquipélagos do nordeste brasileiro (Alves et al. 2004b, Both \& Freitas 2001), bem como em outras regiões oceânicas do mundo, em ambientes com pouca influência antrópica (Murphy 1936, Dorward 1962, Nelson 1980, Harrison et al. 1984). Enquanto que, no litoral do Rio de Janeiro (Coelho et al. 2004), Paraná (Krul 2004) e Santa Catarina (Bege \& Pauli 1989, Branco 2001) e dados do presente estudo, a presença de espécies demersais e bentônicas como $P$. brasiliensis, I. parvipinnis, C. striatus, Stellifer, Porychthis porosissimus (Valenciennes, 1837) e Trachurus lathami Nichols, 1920, de-

Tabela I. Composição da dieta de jovens e adultos de S. leucogaster, representada por número de regurgitos ( $\mathrm{N})$, número médio de espécies por regurgito (Nsp), número de presas (NPr), e amplitudes de comprimento e biomassa com as respectivas médias e desvio padrão $(M \pm d p)$.

\begin{tabular}{|c|c|c|c|c|c|c|c|c|c|c|}
\hline \multirow{2}{*}{ Estágio } & \multirow{2}{*}{$N$} & \multirow{2}{*}{ Nsp } & \multicolumn{4}{|c|}{ Comprimento Padrão $(\mathrm{cm})$} & \multicolumn{4}{|c|}{ Biomassa (g) } \\
\hline & & & NPr & $<$ & $>$ & $M \pm d p$ & $<$ & $>$ & $M \pm d p$ & Total \\
\hline Adulto & 222 & 1,5 & 3,2 & 2,0 & 39,0 & $13,1 \pm 4,4$ & 0,3 & 198,5 & $29,0 \pm 24,8$ & $22.788,0$ \\
\hline Jovem & 35 & 1,4 & 3,3 & 4,0 & 19,4 & $11,8 \pm 3,2$ & 1,7 & 120,1 & $24,6 \pm 20,2$ & $3.961,6$ \\
\hline
\end{tabular}


Tabela II. Relação das espécies presentes nos regurgitos de $S$. leucogaster, quanto ao habitat das presas $(\mathrm{P}=$ pelágico, $\mathrm{D}=$ demersal, $\mathrm{B}=$ bentônico), comprimento médio $(\mathrm{cm})$, biomassa média $(\mathrm{g})(\mathrm{M} \pm \mathrm{dp}=$ média \pm desvio padrão) e índice de importância relativa (IRI).

\begin{tabular}{|c|c|c|c|c|c|c|c|c|}
\hline \multirow{2}{*}{ Taxon } & \multirow{2}{*}{ Habitat } & \multicolumn{2}{|c|}{ Número } & \multirow{2}{*}{$\frac{\text { Comprimento }}{\mathrm{M} \pm \mathrm{dp}(\mathrm{cm})}$} & \multicolumn{3}{|c|}{ Biomassa (g) } & \multirow{2}{*}{ IRI } \\
\hline & & Regurgitos & Presas & & $M \pm d p$ & Total & $\%$ & \\
\hline \multicolumn{9}{|l|}{ Mollusca } \\
\hline \multicolumn{9}{|l|}{ Loligonidae } \\
\hline Loligo plei Blainville, 1823 & $P$ & 6 & 14 & - & $15,8 \pm 12,3$ & 220,6 & 0,8 & 2,7 \\
\hline Loligo sanpaulenses (Brakoniecki, 1984) & $P$ & 10 & 18 & $5,9 \pm 3,1$ & $13,3 \pm 7,9$ & 238,8 & 0,9 & 4,2 \\
\hline \multicolumn{9}{|l|}{ Crustacea } \\
\hline \multicolumn{9}{|l|}{ Solenoceridae } \\
\hline Pleoticus muelleri (Bate, 1888) & B & 3 & 4 & $4,3 \pm 0,8$ & $0,6 \pm \quad 0,2$ & 2,5 & 0,2 & 0,7 \\
\hline \multicolumn{9}{|l|}{ Osteichthyes } \\
\hline Engraulidae & & 4 & 8 & $10,9 \pm 0,8$ & $11,5 \pm$ & 92,0 & 0,3 & 1,2 \\
\hline Engraulis anchoita Hubbs \& Marine, 1936 & $P$ & 1 & 2 & - & $3,8 \pm 2,7$ & 7,6 & 0,1 & 0,2 \\
\hline Lycengraulis grossidens Agassiz, 1829 & $P$ & 6 & 30 & $7,9 \pm 2,7$ & $9,9 \pm 23,7$ & 298,7 & 1,1 & 5,6 \\
\hline Cetengraulis edentulus (Cuvier, 1829) & $P$ & 27 & 135 & $12,2 \pm 2,4$ & $19,7 \pm 9,7$ & $2.659,5$ & 9,9 & 178,2 \\
\hline Clupeidae & & 2 & 3 & $16,0 \pm 1,4$ & $44,2 \pm 18,1$ & 132,6 & 0,5 & 0,6 \\
\hline Opisthonema oglinum (lesueur, 1818) & B & 1 & 3 & $12,6 \pm 1,1$ & $23,0 \pm 8,8$ & 68,9 & 0,3 & 0,3 \\
\hline Sardinella brasiliensis (Steindachner, 1789) & $P$ & 4 & 8 & $12,2 \pm 2,1$ & $18,2 \pm 16,4$ & 145,7 & 0,5 & 1,4 \\
\hline Pellona harroweri (Fowler, 1917) & $P$ & 4 & 13 & $9,8 \pm 3,4$ & $11,7 \pm 10,9$ & 152,8 & 0,6 & 1,8 \\
\hline \multicolumn{9}{|l|}{ Synodontidae } \\
\hline Synodus intermedius (Spix\&Agassiz, 1829) & B & 1 & 1 & - & - & 75,6 & 0,3 & 0,2 \\
\hline \multicolumn{9}{|l|}{ Phycidae } \\
\hline Urophycis brasiliensis (Kaup, 1858) & $\mathrm{D}$ & 1 & 1 & - & - & 23,3 & 0,1 & 0,2 \\
\hline \multicolumn{9}{|l|}{ Batrachoididae } \\
\hline Porichthys porosissimus (Valenciennes, 1837) & $\mathrm{D}$ & 28 & 55 & $14,3 \pm 4,1$ & $26,3 \pm 19,1$ & $1.445,0$ & 5,4 & 44,1 \\
\hline \multicolumn{9}{|l|}{ Mugilidae } \\
\hline Mugil curema Valenciennes, 1836 & $\mathrm{D}$ & 4 & 16 & $13,4 \pm 2,0$ & $21,3 \pm 10,5$ & 341,2 & 1,3 & 3,5 \\
\hline Mugil platanus Günther, 1880 & $\mathrm{D}$ & 1 & 1 & - & - & 56,8 & 0,2 & 0,2 \\
\hline \multicolumn{9}{|l|}{ Hemiramphidae } \\
\hline Hiporhampus unifasciatus (Ranzani, 1842) & B & 1 & 1 & - & $39,9 \pm 11,2$ & 79,8 & 0,3 & 0,2 \\
\hline \multicolumn{9}{|l|}{ Fistularidae } \\
\hline Fistularia tabacaria Linnaeus, 1758 & B & 1 & 1 & - & - & 35,6 & 0,1 & 0,2 \\
\hline \multicolumn{9}{|l|}{ Triglidae } \\
\hline Prionotus punctatus (Bloch, 1797) & $\mathrm{D}$ & 2 & 2 & - & $23,5 \pm$ & 30,5 & 0,1 & 0,4 \\
\hline \multicolumn{9}{|l|}{ Serranidae } \\
\hline Diplectrum formosum (Linnaeus, 1766) & B & 1 & 1 & - & - & 13,4 & 0,1 & 0,2 \\
\hline Carangidae & & 7 & 10 & $13,0 \pm 0,9$ & $18,3 \pm$ & 183,0 & 0,7 & 2,3 \\
\hline Trachurus lathami Nichols, 1920 & B & 15 & 35 & $12,0 \pm 2,2$ & $16,3 \pm 7,2$ & 571,8 & 2,1 & 12,8 \\
\hline Trachinotus falcatus (Linnaeus, 1758) & B & 3 & 3 & $14,2 \pm 0,2$ & $47,5 \pm$ & 142,4 & 0,5 & 0,8 \\
\hline \multicolumn{9}{|l|}{ Gerreidae } \\
\hline Eucinostomus argenteus Baird\&Girard, 1855 & B & 1 & 1 & - & - & 64,4 & 0,2 & 0,2 \\
\hline Haemulidae & & 2 & 5 & $12,4 \pm 2,8$ & $30,3 \pm 16,4$ & 151,6 & 0,6 & 0,8 \\
\hline Conodon Nobilis (Linnaeus, 1758) & $\mathrm{D}$ & 1 & 1 & - & - & 43,5 & 0,2 & 0,2 \\
\hline Scianidae & & 32 & 53 & $12,6 \pm 2,2$ & $30,6 \pm 21,9$ & $1.623,7$ & 6,1 & 48,1 \\
\hline Ctenosciaena gracilicirrhus (Metzelaar, 1919) & $\mathrm{D}$ & 3 & 7 & $14,8 \pm 1,5$ & $44,8 \pm 14,0$ & 313,8 & 1,2 & 1,7 \\
\hline Paralonchurus brasiliensis (Steindachner, 1875) & $\mathrm{D}$ & 75 & 129 & $15,4 \pm 3,4$ & $46,2 \pm 29,0$ & $5.966,8$ & 22,3 & 385,6 \\
\hline
\end{tabular}


Tabela II. Continuação.

\begin{tabular}{|c|c|c|c|c|c|c|c|c|}
\hline \multirow{2}{*}{ Taxon } & \multirow{2}{*}{ Habitat } & \multirow{2}{*}{\multicolumn{2}{|c|}{$\frac{\text { Número }}{\text { Regurgitos Presas }}$}} & \multirow{3}{*}{$\begin{array}{c}\frac{\text { Comprimento }}{\mathrm{M} \pm \mathrm{dp}(\mathrm{cm})} \\
13,3 \pm 0,8\end{array}$} & \multicolumn{3}{|c|}{ Biomassa (g) } & \multirow{3}{*}{$\frac{\mid \mathrm{IRI}}{3,1}$} \\
\hline & & & & & \multirow{2}{*}{$\frac{M \pm d p}{42,7 \pm 22,9}$} & \multirow{2}{*}{$\frac{\text { Total }}{384,5}$} & \multirow{2}{*}{$\frac{\%}{1,4}$} & \\
\hline Micropogonias furnieri (Desmarest, 1823) & $\mathrm{D}$ & 7 & 9 & & & & & \\
\hline Larimus breviceps Cuvier, 1830 & $\mathrm{D}$ & 1 & 1 & - & - & 56,0 & 0,2 & 0,2 \\
\hline Cynoscion striatus (Cuvier, 1829) & $\mathrm{D}$ & 37 & 64 & $13,5 \pm 2,7$ & $28,1 \pm 17,4$ & $1.796,5$ & 6,7 & 63,0 \\
\hline Isopisthus parvipinnis (Cuvier, 1830) & $\mathrm{D}$ & 38 & 65 & $13,4 \pm 2,8$ & $27,8 \pm 17,4$ & $1.805,6$ & 6,8 & 64,4 \\
\hline Stellifer rastrifer (Jordan, 1889) & $\mathrm{D}$ & 4 & 10 & $13,1 \pm 2,7$ & $26,9 \pm 14,6$ & 268,6 & 1,0 & 2,1 \\
\hline Stellifer brasiliensis (Schultz, 1945) & $\mathrm{D}$ & 1 & 1 & - & - & 72,0 & 0,3 & 0,2 \\
\hline Stellifer & & 18 & 34 & $11,6 \pm 2,4$ & $28,1 \pm 22,8$ & 953,7 & 3,6 & 19,4 \\
\hline \multicolumn{9}{|l|}{ Mullidae } \\
\hline Mullus argentinae Hubbs \& Marini, 1933 & $\mathrm{D}$ & 1 & 2 & $17,0 \pm 2,8$ & $33,0 \pm 20,9$ & 65,9 & 0,2 & 0,3 \\
\hline \multicolumn{9}{|l|}{ Trichiuridae } \\
\hline Trichiurus lepturus Linnaeus, 1758 & $\mathrm{D}$ & 12 & 18 & $28,8 \pm 7,2$ & $29,0 \pm 29,0$ & 522,6 & 2,0 & 7,0 \\
\hline \multicolumn{9}{|l|}{ Scombridae } \\
\hline Thunnus obesus (Lowe, 1839) & $\mathrm{P}$ & 1 & 1 & - & - & 198,5 & 0,7 & 0,3 \\
\hline \multicolumn{9}{|l|}{ Cynoglossidae } \\
\hline $\begin{array}{l}\text { Symphurus tesselatus (Quoyd \& Gaimard, } \\
\text { 1824) }\end{array}$ & $\mathrm{D}$ & 5 & 10 & $14,2 \pm 4,1$ & $29,1 \pm 17,9$ & 291,4 & 1,1 & 2,5 \\
\hline Balistidae & & 1 & 1 & - & - & 28,6 & 0,1 & 0,2 \\
\hline \multicolumn{9}{|l|}{ Diodontidae } \\
\hline Cyclichthys spinosus (Linnaeus, 1758) & $\mathrm{D}$ & 1 & 1 & - & - & 36,3 & 0,1 & 0,2 \\
\hline Bolo (material não identificado) & & 102 & - & - & $49,9 \pm 40,6$ & $5.087,4$ & 19,0 & \\
\hline Total & & 476 & 100 & & & $26.749,6$ & 100,0 & \\
\hline
\end{tabular}

monstram a influência das atividades pesqueiras como disponibilizadoras desses recursos não tradicionais na dieta do atobá-marrom.

Nos arquipélagos de Abrolhos e São Paulo e São Pedro, $S$. leucogaster explorou sete e nove espécies de peixes, onde os integrantes da família Exocoetidae contribuíram com 84,6\% e 93,0\% das presas consumidas, respectivamente (Alves et al. 2004b, Вотн \& Freitas 2001). Já na região de Arraial do Cabo (Rio de Janeiro), utilizou 34 espécies, com predomino dos peixes Batrachoididae (29,6\%) (Coelho et al. 2004), nos Arquipélagos de Currais (PR), com 33 espécies (Krul 2004), e Moleques do Sul, com 35 espécies, onde os peixes da família Sciaenidae contribuíram com 30,7 e $28,8 \%$, respectivamente. Essas variações na diversidade dos itens predados, principalmente nas ilhas costeiras do litoral brasileiro, provavelmente estão associadas aos eventos do ciclo de vida e abundância das presas vulneráveis a pesca de arrasto nas proximidades dos sítios de nidificação (Branco 2001, KRUL 2004).

O tamanho dos peixes consumidos pelas aves marinhas está diretamente relacionado ao porte das aves e a disponibilidade de presas (BRANCO 2001). O comprimento médio e biomassa das presas, nos regurgitos de $S$. leucogaster nas Ilhas Moleques do Sul foram superiores aos registrados no litoral do Paraná $(10,4 \pm$ 4,7 cm e 18,6 \pm 27,7 g) (KRUL 2004), e Armação do Itapocoroy, Penha, Santa Catarina (16,5 $\pm 4,2 \mathrm{~cm})$ (Branco 2001). Enquanto que, em São Pedro e São Paulo (16,2 \pm 1,6 cm e 132,1 \pm 17,0 g) (Both \& Freitas 2001) e Abrolhos (13,9 cm) (Alves et al. 2004b), as presas foram relativamente maiores que dessas regiões costeiras, provavelmente em função da baixa atividade pesqueira e o porte dos peixes disponíveis em ilhas oceânicas. Por outro lado, em Rose Atoll, Samoa (arquipélago do Havaí) as amostras variaram de 2,9 a 10,3 cm, com média de 6,4 cm (Harrison et al. 1984).

Segundo Alves et al. (2004b), os maiores valores de IRI na dieta do atobá-marrom de Abrolhos reforçam a importância dos peixes Exocoetidae $(11.447,79)$ e Clupeidae $(851,37)$, corroborando o obtido em Moleques do Sul para os Sciaenidae, Engraulidae e Batrachoididae. Porém, a concentração de aves em uma determinada área pode causar a diminuição dos recursos alimentares, influindo diretamente no sucesso reprodutivo das mesmas (Ashmole 1971, Shreiber \& Burger 2001).

Sula leucogaster nidifica ao longo do ano nas Ilhas Moleques do Sul, limite austral de ocorrência de colônias de reprodução desta espécie, sendo que a maioria dos ninhos com ovos e filhotes foram observados entre junho a março (BRANCO 2004). Assim, o esforço reprodutivo pode ser utilizado como um indicador da disponibilidade de alimento nas proximidades da colônia, nos meses de inverno, primavera e verão. Essa oferta de presas, principalmente das demersais e bentônicas reforçam a contribuição da pesca camaroeira no litoral catarinense; onde 
peixes que normalmente não ocorrem na dieta dos atobás, tornaram-se disponíveis através do descarte da ictiofauna acompanhante nesta arte de pesca (Branco 2001).

\section{AGRADECIMENTOS}

À Universidade do Vale do Itajaí, através do Centro de Ciências Tecnológicas, da Terra e do Mar - CTTMar, pelas facilidades colocadas à disposição. À Fundação do Meio Ambiente, de Santa Catarina - FATMA, pelas autorizações concedidas para trabalhar nas Ilhas Moleques do Sul. Aos orientados e estagiários: Cristiano Lombardo Evangelista, Juliano César Hillesheim e Felipe Freitas Junior entre outros, pelo valioso auxílio nos trabalhos de campo e triagem do material.

\section{REFERÊNCIAS BIBLIOGRÁFICAS}

Alves, V.S.; A.B.A. Soares \& G.S. Couto. 2004a. Aves marinhas e aquáticas das ilhas do estado do Rio de Janeiro, p. 83-100. In: J.O. BRANCO (Ed.). Aves marinhas e insulares brasileiras: biologia e conservação. Itajaí, Editora da Univali, 266p.

Alves, V.S.; A.B.A. SoAres; G.S. Couto; M.A. Efe \& A.B.B. 2004b. Aves marinhas de Abrolhos, p.213-232. In: J.O. Branco (Ed.). Aves marinhas e insulares brasileiras: biologia e conservação. Itajaí, Editora da Univali, 266p.

Ashmole, N.P. 1971. Seabird ecology and the marine environment, p. 223-287. In: D.S. Farner \& J.S. King (Ed.). Avian Biology. New York, Academic press, vol. 1, 643p.

Baltz, D.M. \& G.V. Morejohn. 1977. Food habits and niche overlap of seabirds wintering on Monterey Bay, Califórnia. Auk, Fayetteville, 94: 527-543.

Bege, L.A.R. \& B.T. PAuli. 1989. As aves nas Ilhas Moleques do Sul - Santa Catarina: Aspectos da ecologia, etologia e anilhamento de aves marinhas. Florianópolis, Fatma, 64p.

Both, R. \& T.R.O. Freitas. 2001. A dieta de Sula leucogaster, Anous stolidus e Anous minutus no Arquipélago de São Pedro e São Paulo, Brasil, p. 313- 337. In: J.B. Albuquerque; J.F. Candido Jr.; F.C. Straube \& A.L. Roos (Eds). Ornitologia e Consevação: da ciência às estratégias. Tubarão, Editora Unisul, 341p.

Bотн, R. \& T.R.O. Freitas. 2004. Aves marinas no Arquipélago de São Pedro e São Paulo, p. 193-212. In: J.O. Branco (Ed.). Aves marinhas e insulares brasileiras: biologia e conservação. Itajaí, Editora da Univali, 266p.

BRANCO, J.O. 2001. Descartes da pesca do camarão sete-barbas como fonte de alimento para aves marinhas. Revista Brasileira de Zoologia, Curitiba, 18 (1): 293-300.

Branco, J.O. 2004. Aves marinhas das Ilhas de Santa Catarina, p.15-36. In: J.O. Branco (Ed.). Aves marinhas e insulares brasileiras: biologia e conservação. Itajaí, Editora da Univali, 266p.

CAIrns, D.K. 1987. Seabirds as Indicators of Marine Food Supplies. Biological Oceanography, Toronto, 5: 261-271.

CaIRns, D.K. 1992. Bridging the gap between ornithology and fisheries science: Use of seabird data in stock assessment models. Condor, San Francisco, 94: 811-824.

Coelno, E.P.; V.S. Alves; A.B.A. Soares; G.S. Couto; M.A. Efe \& A.B.B. Ribeiro. 2004. O atobá marrom (Sula leucogaster) na ilha de Cabo Frio, Arraial do Cabo, Rio de Janeiro, Brasil, p. 233-254. In: J.O. BRANCO (Ed.). Aves marinhas e insulares brasileiras: biologia e conservação. Itajaí, Editora da Univali, 266p.

Diamond, A.W. 1983. Feeding overlap in some tropical and temperate seabird communities. Avian Biology, New York, 8: 24-46.

DORWARD, D.F. 1962. Comparative biology of the white booby and the brown booby Sula app. in Atascension. Ibis, London, 10 (1): 79-82.

Figueiredo, J.L. \& N. Menezes. 1978. Manual de peixes marinhos do Sudeste do Brasil. II. Teleostei (1). São Paulo, Museu de Zoologia, Universidade de São Paulo, 110p.

Furness, R.W. \& K.C.J. CAMPhuysen. 1997. Seabirds as monitors of the marine environment. ICES Journal of Marine Science, Coral Gables, 54: 726-737.

Furness, R.W. \& J. CoOper. 1982. Interactions between breeding seabird and pelagic fish populations in the southtern Benguela region. Marine Ecology Progress Series, Nordbunte, 8: 243-250.

Furness, R.W. \& P. Monaghan. 1987. Seabird Ecology. New York, Chapman and Hall, 164p.

Garthe, S.; K.C.J. Camphuysen \& R.W. Furness. 1996. Amounts of discards by commercial fisheries and their significance as food for seabirds in the North Sea. Marine Ecology Progress series, Nordbunte, 136: 1-11.

HARRISON, C.S.; T.S. HILDA \& M.P. SEKI. 1984. Interactions between breeding seabirds and pelagic fish populations in the Southern Benguela region. Marine Ecology Program Series, Nordbunte, 8: 243-250.

KRUL, R. 2004. Aves marinhas costeiras do Paraná, p. 37-56 In: J.O. Branco (Ed.). Aves marinhas e insulares brasileiras: biologia e conservação. Itajaí, Editora da Univali, 266p.

Menezes, N. \& J.L. Figueiredo. 1980. Manual de peixes marinhos do Sudeste do Brasil. IV. Teleostei (3). Museu de Zoologia, Universidade de São Paulo, 96p.

Menezes, N. \& J.L. Figueiredo. 1985. Manual de peixes marinhos do Sudeste do Brasil. V. Teleostei (4). São Paulo, Museu de Zoologia. Universidade de São Paulo, 105p.

Montevecchi, W.A.; V.L. Birt \& D.K. Cairns. 1988. Dietary Changes of Seabirds Associated with Local Fisheries Failures. Biological Oceanography, United Kingdom, 5: 153-161.

Murphy, R.C.1936. Oceanic birds of South America. New York, The American Museum of Natural History, vol. 2, 1245p.

Nelson, J.B. 1978. The Sulidae: Gannets and Boobies. Oxford, Oxford University Press, 1012p.

Nelson, J.B. 1980. Seabirds - their biology and ecology. London, The Hamlyn Publishing Group, 224p.

Oro, D. 1996. Effects of trawler discard availability on egg laying and breeding success in the lesser black-backed gull Larus 
fuscus in the western Mediterranean. Marine Ecology Progress series, Nordbunte, 132: 43-46.

PInKas, L.M.S. \& I.L.K. Iverson. 1971. Food habits of albacore, bluefin tuna and bonito in California waters. Fisheries Bulletin, California, 152: 1-105.

Shulz-Neto, A. 2004. Aves marinhas do Atol das Rocas, p. 169192. In: J.O. BRANCO (Ed.). Aves marinhas e insulares brasileiras: biologia e conservação. Itajaí, Editora da Univali, 266p.

Recebido em 11.IV.2005; aceito em 07.XI.2005.
Shreiber, E.A. \& J. Burger. 2001. Biology of marine birds, p. 115. In: E.A. Shreiber \& J. Burger (Ed.). Seabirds in the marine environment. Boca Raton, CRC Press, 722p.

SICK, H. 1997. Ornitologia Brasileira. Rio de Janeiro, Nova Fronteira, 862p.

Stratoudakis, Y. 1999. Recommenations for improving the estimation of number of seabirds potentially sustained by fisheries discards. Marine Ecology Progress series, Nordbunte, 176: 307-309. 